\title{
Spin configuration of ferromagnetic/antiferromagnetic nano-composite particles
}

\author{
Nguyen N. Phuoc ${ }^{* 1}$, Takao Suzuki ${ }^{1}$, Roy W. Chantrell ${ }^{2}$, and Uli Nowak ${ }^{2}$ \\ 1 Information Storage Materials Laboratory, Toyota Technological Institute, 2-12-1 Hisakata, \\ Tempaku, Nagoya 468-8511, Japan \\ 2 Department of Physics, University of York, York YO10 5DD, UK
}

\author{
PACS 75.25.+z, 75.30.Gw, 75.70.Cn, 75.75.+a
}

A systematic study of the spin configuration of ferromagnetic(core)/antiferromagnetic(shell) nanocomposite particles was carried out by two approaches: micromagnetic and atomistic simulations. In the case of strong interlayer exchange coupling between ferromagnet and antiferromagnet, the micromagnetic simulation result is consistent with the atomistic simulation result. However, in the case of weak interlayer exchange coupling the micromagnetic simulation predicts a multi-domain structure of the ferromagnet, which is inconsistent with the atomistic simulation. The failure of micromagnetic simulation in describing the spin structure of the nano-composite particles might be interpreted in terms of the under-estimation of the exchange energy for rapid spatial fluctuations of the magnetization by the continuum exchange formalism or might be due to the cooling process procedure. Also, the variation of spin structure as a function of the uniaxial anisotropy of the core is presented and discussed.

\section{Introduction}

Among many studies on nano-particles [1], exchange coupling in ferromagnetic (FM)-antiferromagnetic (AF) nano-composite particles is an appealing subject but there is still very little work on this theme. The reason for this is possibly because the applications of exchange bias are mostly in thin film form. Recently, however, the study of exchange bias in nano-particles has received much interest due to the potential applications in magnetic recording technology $[2,3]$. From theoretical approach, there have been still few papers addressing exchange bias in nanostructures [4]. Among these few papers, there is no report about the system with high magnetic anisotropy such as FePt. Moreover, in view of the recently proposed idea of using $\mathrm{FePt} / \mathrm{FeRh}$ for heat-assisted magnetic recording technique to increase the storage density [5], the understanding of the exchange coupling between a ferromagnet with high magnetic anisotropy and an antiferromagnet is of crucial importance. The present paper therefore focuses on the study of the exchange coupling in $\mathrm{FePt}(\mathrm{core}) / \mathrm{FeMn}$ (shell) nano-composite particles by two approaches: micromagnetic simulation [6] and atomistic simulation [7].

\section{Models}

The micromagnetic simulation is based on the Landau-Lifshitz-Gilbert equation [8], where the equilibrium state of the spin configuration is obtained by minimization of the total energy from the random state. The equilibrium state, on the other hand, from the atomistic model is obtained by heating the nano-

\footnotetext{
* Corresponding author: e-mail: phuoc@toyota-ti.ac.jp, Phone: 81-52-809-1872, Fax: 81-52-809-1874
} 
particles beyond the Curie temperature and cooling down to $\mathrm{T}=0 \mathrm{~K}$. Another major difference between micromagnetic and atomistic models is that the atomistic approach is based on the calculation at the atomistic level, using a reasonable physical model for the exchange, rather than using the longwavelength (micromagnetic) approximation [9]. In particular, the Heisenberg form of exchange is used in the atomistic model. In other words, although the cell sizes of both micromagnetic and atomistic models look to be the same, the calculation method is totally different. For micromagnetic cells, a constant value of magnetization is taken while in the atomistic model, the magnetization is averaged over all atoms [9].

In this paper, the nano-composite particles considered have a spherical shape with the total diameter of $9.6 \mathrm{~nm}$ and are composed of a FM core with the diameter of $4.8 \mathrm{~nm}$, which is covered by an AF shell. The cell size for simulation is chosen to be $0.4 \mathrm{~nm}$, which is close to the lattice constant of both FePt and FeMn. The core parameters are $\mathrm{K}_{\mathrm{U}}=7 \times 10^{7} \mathrm{erg} / \mathrm{cc}, \mathrm{M}_{\mathrm{S}}=1100 \mathrm{emu} / \mathrm{cc}, \mathrm{J}_{\mathrm{FM}}=4 \times 10^{-14} \mathrm{erg}$ and the shell ones are $\mathrm{K}_{\mathrm{AF}}=1.3 \times 10^{5} \mathrm{erg} / \mathrm{cc}, \mathrm{M}_{\mathrm{S}}=800 \mathrm{emu} / \mathrm{cc}, \mathrm{J}_{\mathrm{AF}}=-1.2 \times 10^{-14} \mathrm{erg}$ [6], where $\mathrm{K}_{\mathrm{U}}$ is the uniaxial magnetic anisotropy of the $\mathrm{FM}, \mathrm{K}_{\mathrm{AF}}$ the uniaxial magnetic anisotropy of the $\mathrm{AF}, \mathrm{M}_{\mathrm{S}}$ the saturation magnetization, $\mathrm{J}_{\mathrm{FM}}$ the exchange integral of the $\mathrm{FM}, \mathrm{J}_{\mathrm{AF}}$ the exchange integral of the FM. The easy axis of both the core and the shell are chosen in the vertical direction. For the investigation of the effect of interlayer coupling, the exchange-coupling constant between FM and AF is varied from $10^{-16}$ to $10^{-14} \mathrm{erg}$. The influence of the uniaxial magnetic anisotropy $\left(\mathrm{K}_{\mathrm{U}}\right)$ of the core on the spin structure is also investigated with $\mathrm{K}_{\mathrm{U}}$ changing from $10^{4} \mathrm{erg} / \mathrm{cc}$ to $7 \times 10^{7} \mathrm{erg} / \mathrm{cc}$.

\section{Results and discussion}

Figure 1 shows representative spin structures of the core/shell particles obtained from micromagnetic simulation with various interlayer exchange-coupling constant. It is of particular interest to see that as the interlayer exchange coupling between FM and AF $\left(\mathrm{J}_{\mathrm{FM}-\mathrm{AF}}\right)$ is less than $0.32 \times 10^{-14} \mathrm{erg}$, the magnetization arrangement of the core break up into domains even the particle size is very small $(4.8 \mathrm{~nm})$. It should be
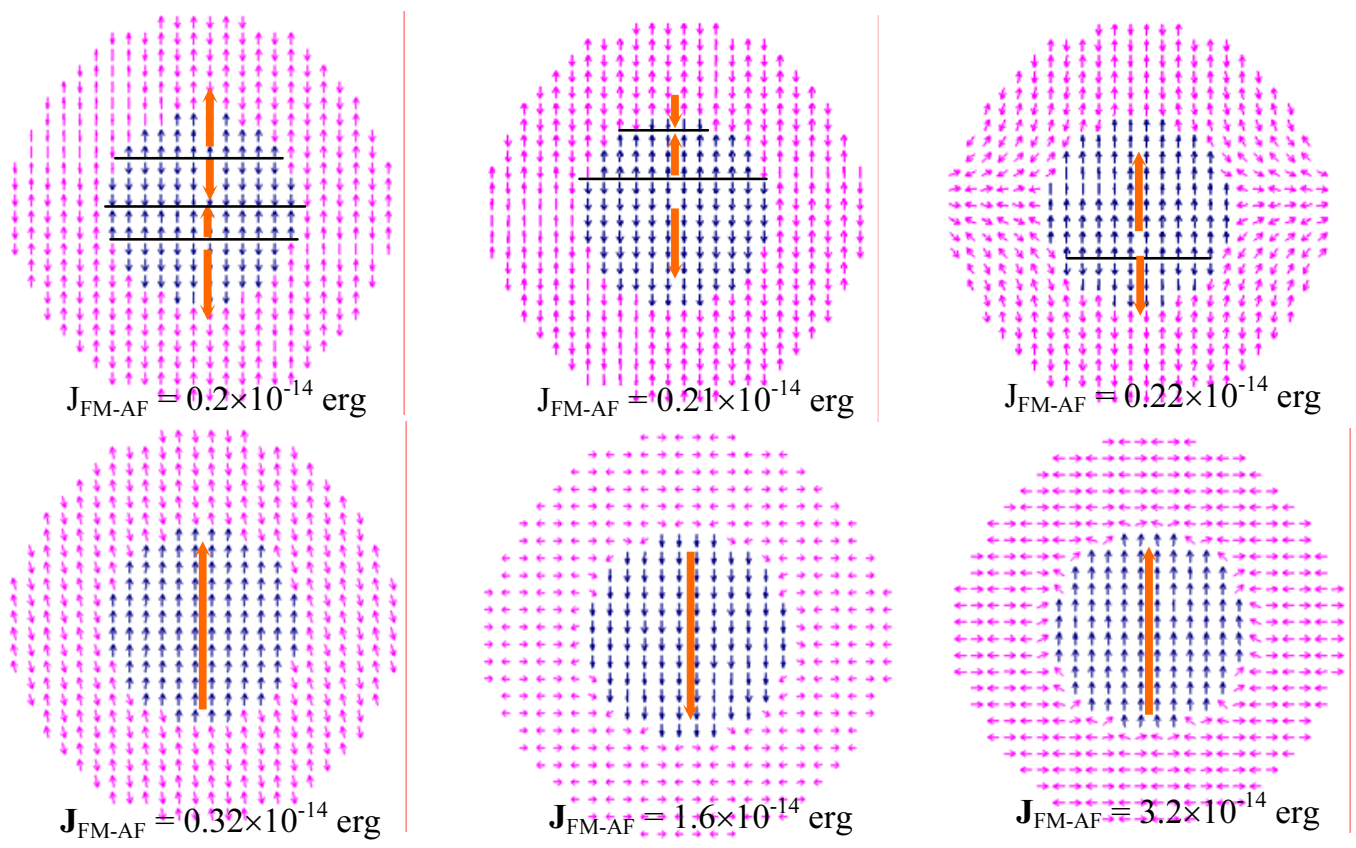

Fig. 1 Spin configurations obtained from micromagnetic simulation in demagnetization state of the FM/AF nanocomposite particles with various interlayer exchange coupling constants. 


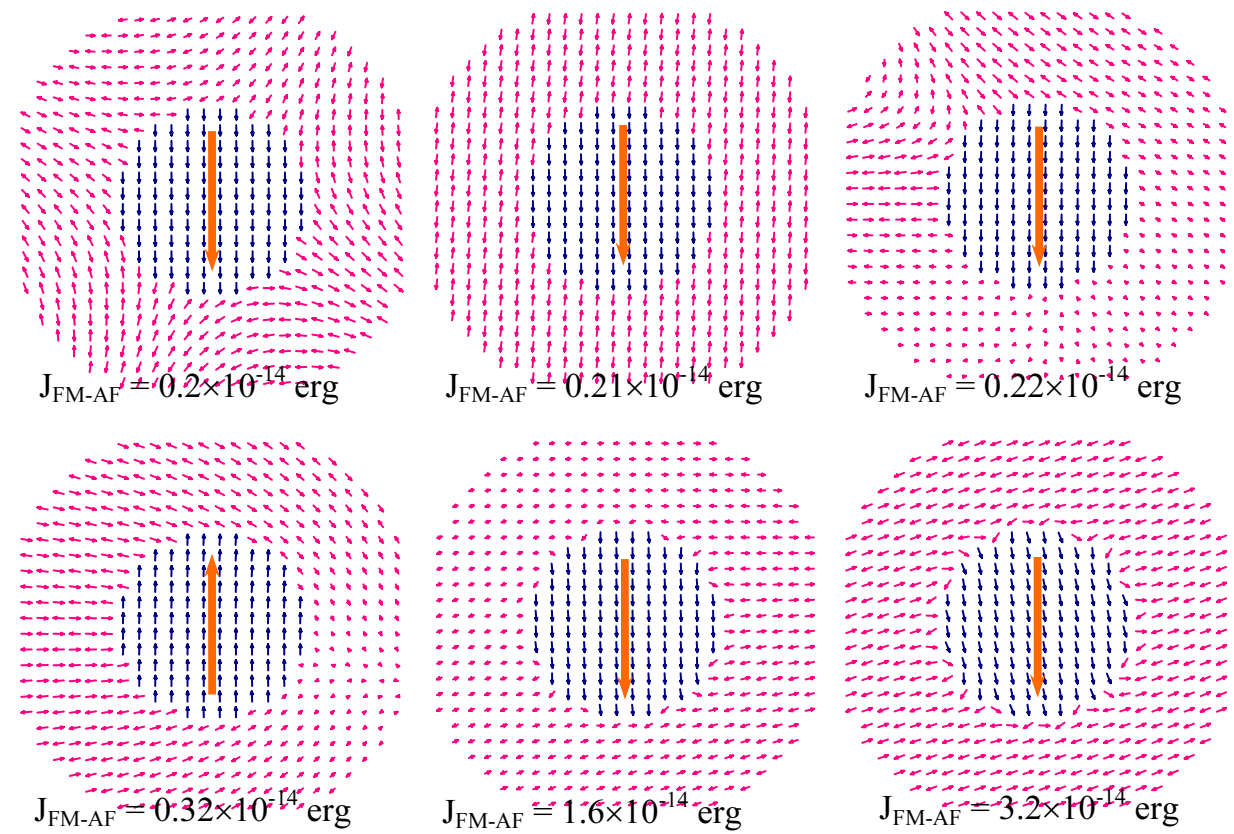

Fig. 2 Spin configurations obtained from atomistic simulation in demagnetization state of the FM/AF nanocomposite particles with various interlayer exchange coupling constants.

noted with such a large anisotropy constant as $\mathrm{K}_{\mathrm{U}}=7 \times 10^{7} \mathrm{erg} / \mathrm{cc}$, the critical size for single domain particle is of the order of $100 \mathrm{~nm}$. Therefore, the formation of multiple-domain in this case is unreasonable as it costs a lot of exchange energy in FM system. For stronger interlayer coupling, the core becomes single-domain. Such a behavior cannot be observed if using atomistic simulation as shown in Fig. 2. As in Fig. 2, the single-domain structure is observed in all the cases. It was reported previously similar failure of the micromagnetic simulation in describing the domain structure and the switching behavior of the hard/soft layers when the interlayer exchange coupling is small [7,9]. The reason for this failure is possibly due to the under-estimation of the exchange energy for rapid spatial fluctuations of the magnetization by the (continuum) exchange formalism [9]. Another reason for this might be due to the cooling procedure, where the micromagnetic model does not really cool the system and might get trapped in unphysical states while the spin model does really a cooling, i.e. a slow lowering of temperature. It is also interesting to see that for strong interlayer exchange coupling, the spins of the AF at the interface are flopped due to the effect of the strong coupling with FM and this is observed for both micromagnetic and atomistic simulations. The result therefore implies that micromagnetic simulation with the cell size close to atomic length can only be applied in the case of strong exchange coupling.

Shown in Fig. 3 is the spin configuration of the core/shell particles with the variation of $\mathrm{K}_{\mathrm{U}}$, where the interlayer coupling is fixed with $\mathrm{J}_{\mathrm{FM}-\mathrm{AF}}=4 \times 10^{-14} \mathrm{erg}$. It should be noted that with this strong interlayer coupling, the spin structure obtained from both micromagnetic and atomistic models generally looks similar although there is a minor difference. This confirms the above argument that micromagnetic simulation is valid in the case of strong interlayer exchange coupling. Of interest in Fig. 3 is the changing of the easy axis of the core from horizontal to vertical direction as $\mathrm{K}_{\mathrm{U}}$ increases. This can be explained by assuming that the spins of AF and FM favor to be perpendicular in the case of compensated AF as calculated by Koon [10]. Thus when $\mathrm{K}_{\mathrm{U}}$ is small, the easy axis of the core is forced to be perpendicular to that of the shell. When $\mathrm{K}_{\mathrm{U}}$ and $\mathrm{K}_{\mathrm{AF}}$ are comparable, the strong coupling causes the FM spin tilted. When $\mathrm{K}_{\mathrm{U}}$ is large, then the core anisotropy dominates, causing the spins of the core to be in vertical direction. 


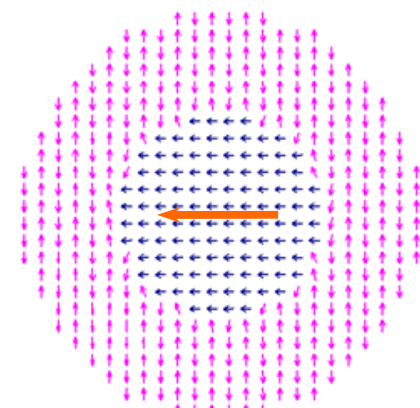

$\mathrm{K}_{\mathrm{U}}=10^{5} \mathrm{erg} / \mathrm{cc}$

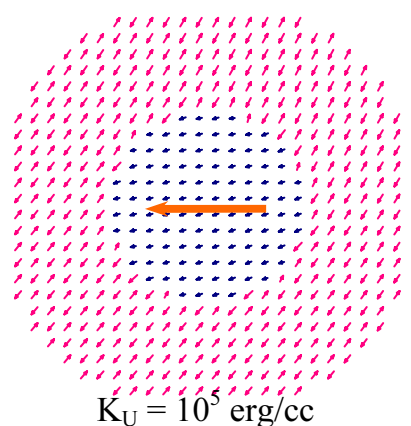

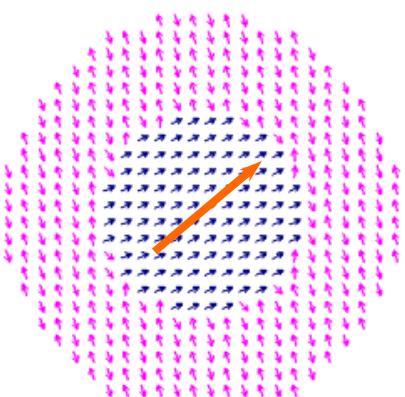

$\mathrm{K}_{\mathrm{U}}=2 \times 10^{7} \mathrm{erg} / \mathrm{cc}$

$\because: 3::^{\prime}:{ }^{\prime}$

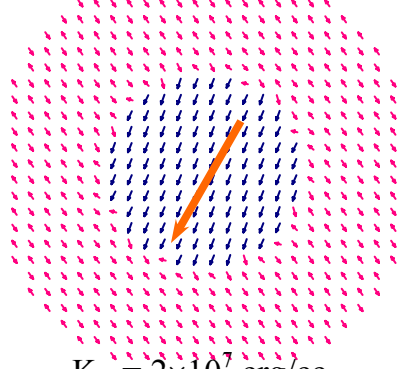

$\mathrm{K}_{\mathrm{U}} \stackrel{\cdots}{=} 2 \times 10^{7} \mathrm{erg} / \mathrm{cc}$
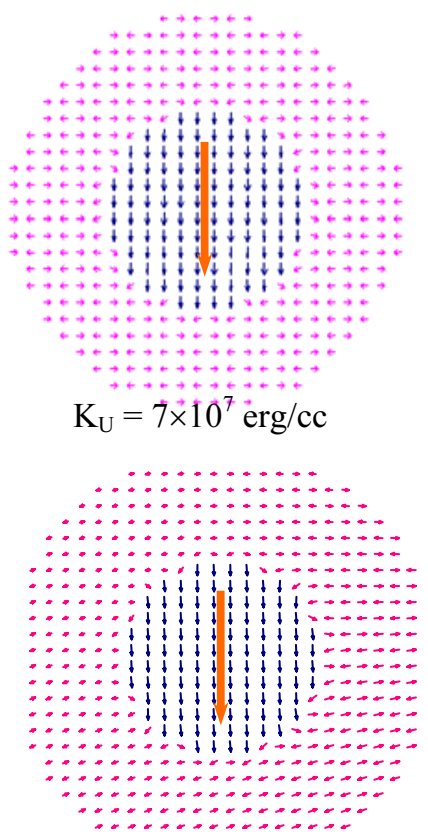

$\mathrm{K}_{\mathrm{U}}^{*}=7 \times 10 \mathrm{erg} / \mathrm{cc}$

Fig. 3 Spin configurations with various $\mathrm{K}_{U}$ obtained from micromagnetic simulation (upper row) and atomistic simulation (lower row) in demagnetization state of the FM/AF nano-composite particles.

\section{Conclusion}

In summary, this paper presents a formation of multiple-domain in the core(FM)/shell(AF) nanoparticles obtained from micromagnetic simulation, which cannot be observed in atomistic model, showing a clear evidence of the failure of micromagnetic simulation in the case of weak interlayer coupling. The micromagnetic model should be applied with caution when the cell size is close to atomic length.

Acknowledgements This work was partially supported by the Academic Frontier Center by MEXT HAITEKU (2004-2008), the Japanese Storage Research Consortium and the Grant-in-Aid for Scientific Research (A) (\#14205049) by the Japanese Ministry of Education, Culture, Sports, Science and Technology.

\section{References}

[1] Q.Y. Xu, Y. Kageyama, and T. Suzuki, IEEE Trans. Magn. 41, 3379 (2005).

[2] J. Nogues et al., Phys. Rep. 422, 65 (2005).

[3] V. Skumryev et al., Nature 423, 850 (2003).

[4] O. Iglesias, X. Batlle, and A. Labarta, Phys. Rev. B 72, 212401 (2006).

[5] J.U. Thiele, S. Maat, and E.E. Fullerton, Appl. Phys. Lett. 82, 2859 (2003).

[6] N.N. Phuoc and T. Suzuki, J. Appl. Phys. 101, 09E501-1 (2007).

[7] F. Garcia-Sanchez, O. Chubykalo-Fesenko, O. Mryasov, and R.W. Chantrell, Physica B 372, 328 (2006).

[8] http://llgmicro.home.mindspring.com/

[9] F. Garcia-Sanchez, O. Chubykalo-Fesenko, O. Mryasov, and R.W. Chantrell, J. Magn. Magn. Mater. 303, 282 (2006).

[10] N.C. Koon, Phys. Rev. Lett. 78, 4865 (1997). 